\title{
High Molecular Weight Spectral Interferences in Mass Spectra of Dissolved Organic Matter
}

\author{
Claudia Patriarca and Jeffrey A. Hawkes*
}

Cite This: J. Am. Soc. Mass Spectrom. 2021, 32, 394-397

Read Online

ABSTRACT: In this paper we investigate interferences that appear in molecular mass spectra from aquatic samples. The interferences are identified as doubly charged ions originating from high molecular weight material, which is especially abundant in terrestrial samples. The interferences could be incorrectly assigned to singly charged formulas with high aromaticity and heteroatom content, as the mass error from such formulas can be less than $1 \mathrm{ppm}$. We propose a strategy for filtering the interference peaks from mass lists based on the presence of their equivalent isotopologue peaks at mass defects of $\sim 0.5 \mathrm{Da}$.

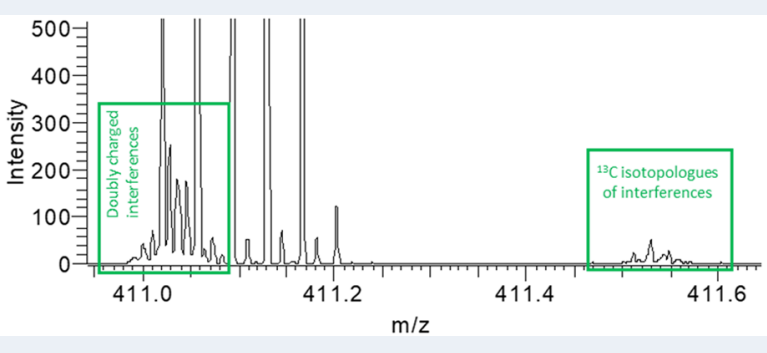

\section{INTRODUCTION}

High-resolution mass spectrometry (HRMS) gives detailed molecular insight into complex environmental mixtures of dissolved organic matter (DOM). Samples are generally ionized by electrospray (ESI) in negative mode to take advantage of the prevalence of carboxylic acid groups in DOM. The resulting mass spectra are processed in routines that remove noise, calibrate the $\mathrm{m} / \mathrm{z}$ axis, and assign formulas. In general, researchers attempt to assign formulas to as much signal as possible, keeping a close control on the allowed constraints in mass accuracy and other metrics such as isotope patterns, in order to minimize false positive assignments.

One critical assumption that has gradually gained acceptance is that the vast majority of signals generated by ESI of the DOM samples are from singly charged ions, ${ }^{1,2}$ although doubly charged ions have been observed in DOM samples by ultrahigh resolution MS and ion mobility MS. ${ }^{3,4}$ The low but noticeable signal from doubly charged species can be erroneously assigned to singly charged formulas if the resolving power is not sufficient, with important implications for how samples are described. Improved insights into the nature of complex environmental samples have been obtained through the detailed separation of mixtures using chromatography, ${ }^{5-}$ and we have found that the doubly charged spectral interferences in the DOM samples can be removed by fractionating the sample with size exclusion chromatography. The implication of this result is that the interferences are not an artifact of the measurement (e.g., doubly charged versions of the same molecules that form singly charged ions) but are generated from separate molecular entities in the sample. The doubly charged "interferences" can be attributed to partial ionization of higher molecular weight DOM, which is of greater abundance in more terrestrially dominated, lightattenuating samples. ${ }^{8}$

We do not recommend that every sample should be fractionated prior to the HRMS analysis but, instead, suggest that formula assignment routines are carefully tuned so that these interferences are not incorrectly assigned, and we also recommend that mass spectrometrists take care to manually inspect mass spectra even as we increasingly employ automated routines to process samples.

\section{METHODS}

Suwannee River Fulvic acid (SRFA) was obtained from the International Humic Substances Society, and it was prepared to $10.3 \mathrm{mg} / \mathrm{mL}$ in $50 \%$ methanol (liquid chromatography-mass spectrometry (LCMS) grade LiChroSolv, VWR) in ultrapure water (Milli-Q Millipore). The sample was fractionated into four size fractions as described in a previous paper, ${ }^{8}$ and further details can be found in the Supporting Information. Briefly, two fractions (high and low molecular weight) were collected after separation by high-pressure size-exclusion chromatography, and they were subsequently analyzed by reversed-phase high-pressure liquid chromatography (HPLC), along with unfractionated SRFA: The two size fractions were equivalent to size ranges of $\sim 1200-2800 \mathrm{Da}$ (Fraction 2 ) and 60-510 Da (Fraction 4) in an equivalent molecular size of poly(styrenesulfonate). ${ }^{9}$ For each of the three samples, HPLCESI-MS data were averaged between 2 and $15 \mathrm{~min}$ to a single mass spectrum and mass list for formula assignment.

Received: September 23, 2020

Revised: November 16, 2020

Accepted: November 16, 2020

Published: November 24, 2020

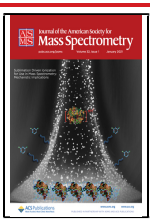




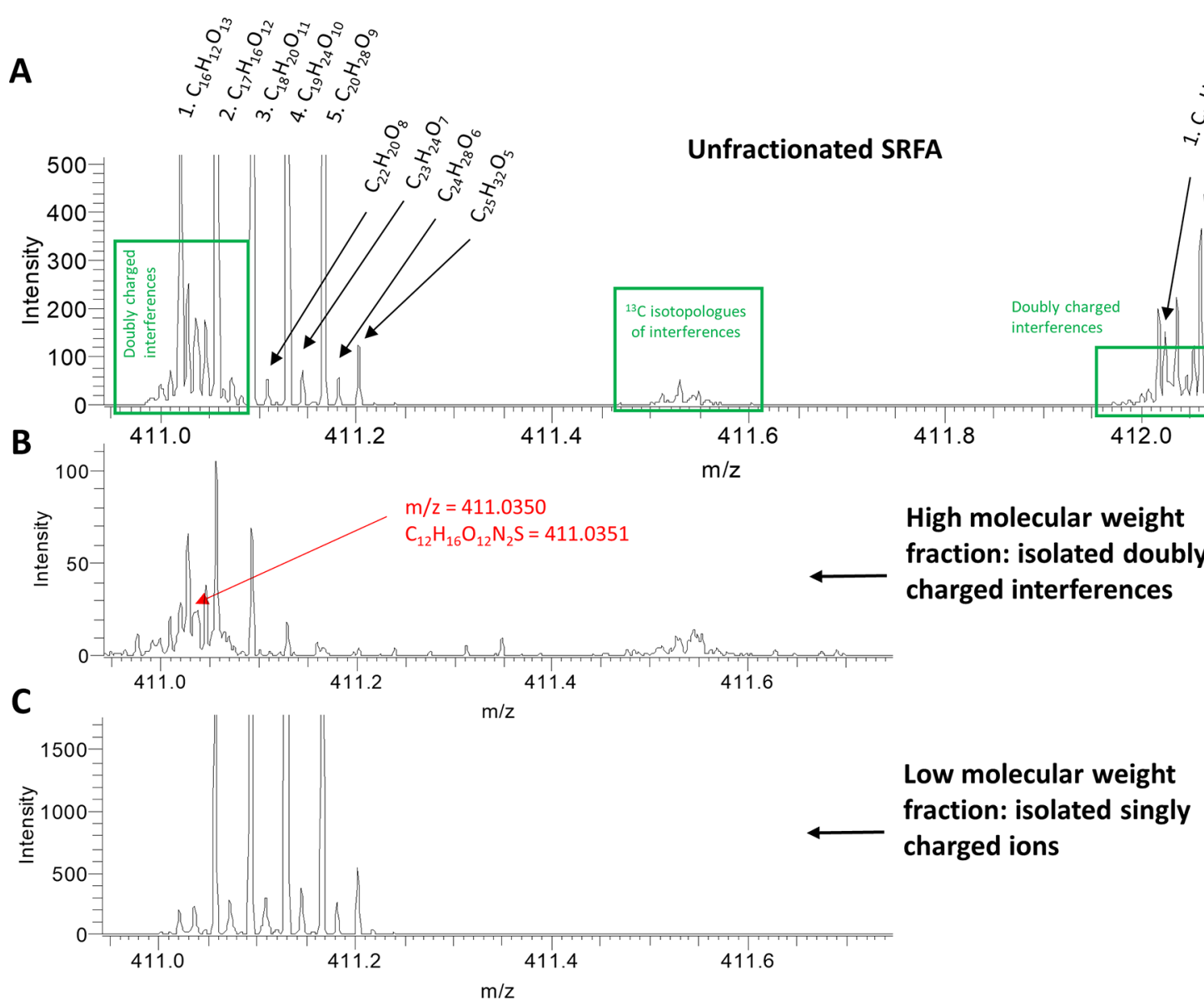

Figure 1. Mass spectra of (A) unfractionated SRFA, (B) high molecular weight fraction, and (C) low molecular weight fraction. Several highly probable formula assignments are indicated in (A) with black text, and suggested doubly charged interferences are indicated in green boxes. An example of a possible false assignment of a likely doubly charged peak is indicated in red in (B). Note that the intensity scale on the $y$-axis is different for each plot, and the high-intensity singly charged ions are cropped in $(A, C)$ in order to display the interferences.
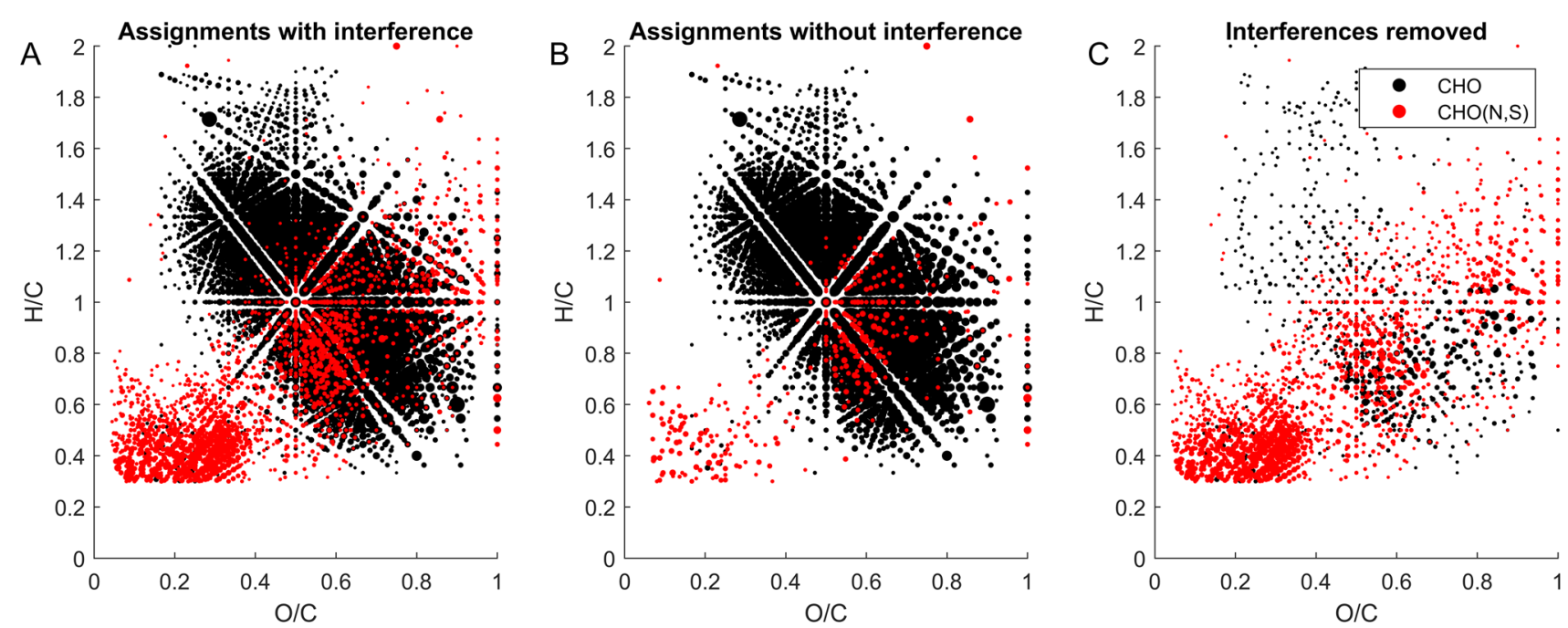

Figure 2. van Krevelen diagrams ( $\mathrm{H} / \mathrm{C}$ vs $\mathrm{O} / \mathrm{C}$ for each assigned formula) for the unfractionated sample. Assignments before (A) and after (B) filtering the data for doubly charged ions. (C) Likely false positive assignments removed. Point size is shown as the square root of intensity; colors indicate $\mathrm{CHO}$ peaks (black) and those with at least one nitrogen or sulfur (red).

\section{RESULTS AND DISCUSSION}

Inspection of a mass window at 411.00-412.25 Da in SRFA showed that some low-intensity doubly charged ions were present (Figure 1). Note that these interferences are present throughout the spectrum, not just at $m / z$ 411. The doubly charged ions have ${ }^{13} \mathrm{C}$ isotopologues at ca. $+0.5 \mathrm{Da}$, while singly charged ions have isotopologues at ca. +1 Da (Figure 1A). Several potential formulas could be assigned to the 
doubly charged ions under some formula assignment algorithm constraints, as indicated in Figure 1B. The intensity of the ${ }^{13} \mathrm{C}$ isotopologues of the doubly charged ions was at the same order of magnitude as the monoisotopic ${ }^{12} \mathrm{C}$ ions, presumably due to the high carbon number of the more likely $\left(\mathrm{M}-2 \mathrm{H}^{2-}\right)$ assignments, which have been made previously at higher resolving power. ${ }^{4}$ This is different than the typical ${ }^{13} \mathrm{C}$ isotopologues of singly charged ions in this mass range (Figure $1 \mathrm{~A})$, which are typically $\sim 10-20 \%$ of the intensity of the monoisotopic ions due to the ions' carbon numbers from 10 to 20 and the natural proportion of ${ }^{13} \mathrm{C} /{ }^{12} \mathrm{C}$ of $\sim 1 \%$.

Fractionation of SRFA into nonadjacent high molecular weight and low molecular weight size fractions demonstrated that the doubly charged ion intensity was due to high molecular weight components of the mixture, and it was not any type of artifact of the ionization and measurement of low molecular weight material (Figure 2). Accordingly, we suggest that this material is genuinely present in the sample and, without time-costly sample fractionation, cannot be removed. Instead the data treatment needs to take the high molecular weight material into account in order to avoid false positive formula assignment. As determined previously, these doubly charged ions are split from some singly charged sulfurcontaining peaks by just $0.43 \mathrm{mDa},{ }^{4}$ leading to the obtained coalescence of ions (Figure 1B) when resolving power is not extremely high. With assumed Gaussian peak shape and $10 \%$ valley definition of resolution, ${ }^{10}$ the mass-dependent peak resolution $(R)$ required to avoid coalescence of these peaks can be calculated from eq 1 .

$$
R=\frac{m / z}{\text { Full Width at Half Maximum }} \geq \frac{2000}{0.43} \mathrm{~m} / z
$$

This relationship is not satisfied at any mass for the Orbitrap used in this study and only for masses less than $\sim 180 \mathrm{~m} / z$ for $12 \mathrm{~T}$ FT-ICR and less than $~ 500$ for $21 \mathrm{~T} \mathrm{FT-ICR,} \mathrm{according} \mathrm{to}$ data from refs 4 and 11 (Figure S1). We found that the high molecular weight material responsible for the spectral interference is more abundant in samples with high terrestrial influence, such as SRFA and headwater stream samples. These species progressively decrease in clearer water aquatic systems and are at the lowest in groundwater and marine water. ${ }^{8}$

There are spectral interferences at each mass (odd and even) at similar intensities, because the typical repeating pattern of odd masses (grouping patterns of $+2 \mathrm{Da}$ ) for singly charged ions is decreased to grouping patterns of $+1 \mathrm{Da}$. This means that the relative abundance of the doubly compared to singly charged ions is higher at even masses (e.g., 412 Da, Figure 1A), leading to a high probability of false positive assignments of nitrogen-containing formulas (Figures 2 and S2).

One approach to avoid false positive assignment of doubly charged ions to singly charged formulas is to simply allow doubly charged formulas. ${ }^{4}$ This would double the processing time of most routines and would lead to some false assignments. Since the vast majority of doubly charged ions are of a very low intensity (as evidenced by the lack of abundant ions at mass defects $0.5-0.6)$ and also coalesce with singly charged peaks, this solution is probably too crude. Interferences greater than $250 \mathrm{Da}$ can only be resolved from singly charged ions by ion mobility spectrometry and greater than or equal to $21 \mathrm{~T}$ Fourier-transform ion cyclotron resonance (FTICR) $\mathrm{MS}^{3,4,12}$ (Figure S1).

In more routine applications, we suggest that interferences should be removed before the noise determination and formula assignment steps. This can be done by identifying the isotopologues of the doubly charged peaks at mass defects of 0.45-0.65 and then removing the equivalent monoisotopic doubly charged peaks at the identified mass of $-0.5017 \mathrm{Da}$ $( \pm 2 \mathrm{ppm})$, which is half the mass of the difference between ${ }^{13} \mathrm{C}$ and ${ }^{12} \mathrm{C}$. This can be done for a mass list in a simple coded loop (see the Supporting Information).

In Figure 2, we show the effect of applying such a filter on the data with van Krevelen diagrams of the assigned formulas for the bulk SRFA sample. Formula assignment tolerance was 1 ppm, and up to two nitrogens and one sulfur were allowed.

It is clear from Figure 2 that a large portion of the doubly charged spectral interferences can be assigned to heteroatomcontaining formulas that occupy the lower left corner of the van Krevelen space and might, therefore, be considered as "condensed aromatics" or "black nitrogen" type of compounds by popular molecular class assignments. These false positive assignments can often be found in the literature in the same surprising corner of the van Krevelen diagram. Simply applying the filter described to remove doubly charged ions from considerations decreased the number of $\mathrm{CHO}+\mathrm{N}, \mathrm{S}$ peaks dramatically from 1892 to 346 .

The numerous high aromaticity false assignments can also be avoided by applying a formula assignment rule (doublebond equivalents minus oxygen less than 10). ${ }^{13}$ Such a rule may still be necessary even after the filter we suggest is applied, as several such assignments were still made after the filter was applied (Figure 2B). In any case, applying such a rule would not remove all false positives we identify in Figure 2C, many of which are found in the center of the plotted van Krevelen space. Improving mass accuracy and isotope pattern requirements might also help, but considering that the spectral interferences lead to many low-intensity ions at every nominal mass (not just odd masses), isotope pattern algorithms may also be confounded.

The best approach should combine noise and interference filtering steps and sensible formula constraints that are adapted according to the resolving power of the instrument used. In any case, all automated routines are likely to make some false negative and positive assignments, and so careful vigilance of the results is required, and expert mass spectrometrists cannot be removed from the process quite yet.

\section{ASSOCIATED CONTENT}

\section{Supporting Information}

The Supporting Information is available free of charge at https://pubs.acs.org/doi/10.1021/jasms.0c00353.

Additional method detail, two supplemental figures and an example MATLAB code for removing spectral interferences from a mass list (PDF)

\section{AUTHOR INFORMATION}

\section{Corresponding Author}

Jeffrey A. Hawkes - Analytical Chemistry, Department of Chemistry BMC, Uppsala University, Uppsala 75237, Sweden; (1) orcid.org/0000-0003-0664-2242;

Email: Jeffrey.hawkes@kemi.uu.se

\section{Author}

Claudia Patriarca - Analytical Chemistry, Department of Chemistry BMC, Uppsala University, Uppsala 75237, Sweden; (1) orcid.org/0000-0002-4301-3923 
Complete contact information is available at:

https://pubs.acs.org/10.1021/jasms.0c00353

\section{Notes}

The authors declare no competing financial interest.

\section{ACKNOWLEDGMENTS}

J.A.H. was funded by the Swedish Research Council (VR Grant No. 2018-04618). The authors thank two reviewers for helping to improve the manuscript.

\section{REFERENCES}

(1) Novotny, N. R.; Capley, E. N.; Stenson, A. C. Fact or artifact: The representativeness of ESI-MS for complex natural organic mixtures. J. Mass Spectrom. 2014, 49, 316-326.

(2) Kujawinski, E. B.; Freitas, M. A.; Zang, X.; Hatcher, P. G.; Green-Church, K. B.; Jones, R. B. The application of electrospray ionization mass spectrometry (ESI MS) to the structural characterization of natural organic matter. Org. Geochem. 2002, 33, 171-180.

(3) Gaspar, A.; Kunenkov, E. V.; Lock, R.; Desor, M.; Perminova, I.; Schmitt-Kopplin, P. Combined utilization of ion mobility and ultrahigh-resolution mass spectrometry to identify multiply charged constituents in natural organic matter. Rapid Commun. Mass Spectrom. 2009, 23, 683.

(4) Smith, D. F.; Podgorski, D. C.; Rodgers, R. P.; Blakney, G. T.; Hendrickson, C. L. 21 T FT-ICR Mass Spectrometer for UltrahighResolution Analysis of Complex Organic Mixtures. Anal. Chem. 2018, 90, 2041-2047.

(5) Woods, G. C.; Simpson, M. J.; Koerner, P. J.; Napoli, A.; Simpson, A. J. HILIC-NMR: Toward the identification of individual molecular components in dissolved organic matter. Environ. Sci. Technol. 2011, 45, 3880-3886.

(6) Hawkes, J. A.; Patriarca, C.; Sjöberg, P. J. R.; Tranvik, L. J.; Bergquist, J. Extreme isomeric complexity of dissolved organic matter found across aquatic environments. Limnol. Oceanogr. Lett. 2018, 3, $21-30$.

(7) Spranger, T.; Pinxteren, D.; Reemtsma, T.; Lechtenfeld, O. J.; Herrmann, H. 2D Liquid Chromatographic Fractionation with Ultrahigh Resolution MS Analysis Resolves a Vast Molecular Diversity of Tropospheric Particle Organics. Environ. Sci. Technol. 2019, 53, 11353-11363.

(8) Patriarca, C.; Balderrama, A.; Može, M.; Sjöberg, P. J. R.; Bergquist, J.; Tranvik, L. J.; Hawkes, J. A. Investigating the ionization of dissolved organic matter by electrospray ionization. Anal. Chem. 2020, 92, 14210.

(9) Hawkes, J. A.; Sjöberg, P. J. R. R.; Bergquist, J.; Tranvik, L. J. Complexity of dissolved organic matter in the molecular size dimension: insights from coupled size exclusion chromatography electrospray ionisation mass spectrometry. Faraday Discuss. 2019, 218, 52-71.

(10) Urban, J.; Afseth, N. K.; Štys, D. Fundamental definitions and confusions in mass spectrometry about mass assignment, centroiding and resolution. TrAC, Trends Anal. Chem. 2014, 53, 126-136.

(11) Hawkes, J. A.; D’Andrilli, J.; Agar, J. N.; Barrow, M. P.; Berg, S. M.; Catalán, N.; Chen, H.; Chu, R. K.; Cole, R. B.; Dittmar, T.; Gavard, R.; Gleixner, G.; Hatcher, P. G.; He, C.; Hess, N. J.; Hutchins, R. H. S.; Ijaz, A.; Jones, H. E.; Kew, W.; Khaksari, M.; Palacio Lozano, D. C.; Lv, J.; Mazzoleni, L. R.; Noriega-Ortega, B. E.; Osterholz, H.; Radoman, N.; Remucal, C. K.; Schmitt, N. D.; Schum, S. K.; Shi, Q.; Simon, C.; Singer, G.; Sleighter, R. L.; Stubbins, A.; Thomas, M. J.; Tolic, N.; Zhang, S.; Zito, P.; Podgorski, D. C. An international laboratory comparison of dissolved organic matter composition by high resolution mass spectrometry: Are we getting the same answer? Limnol. Oceanogr.: Methods 2020, 18, 235.

(12) Leyva, D.; Jaffe, R.; Fernandez-Lima, F. Structural Characterization of Dissolved Organic Matter at the Chemical Formula Level Using TIMS-FT-ICR MS/MS. Anal. Chem. 2020, 92, 11960-11966.
(13) Herzsprung, P.; Hertkorn, N.; Von Tumpling, W.; Harir, M.; Friese, K.; Schmitt-Kopplin, P. Understanding molecular formula assignment of Fourier transform ion cyclotron resonance mass spectrometry data of natural organic matter from a chemical point of view. Anal. Bioanal. Chem. 2014, 406, 7977-7987. 